\title{
Control of magnetic contrast with nonlinear magneto-plasmonics
}

SUBJECT AREAS:

OPTICAL SENSORS

NANOPHOTONICS AND

PLASMONICS

Received

4 March 2014

Accepted

7 August 2014

Published

26 August 2014

Correspondence and requests for materials should be addressed to W.Z. (wzheng@email. wm.edu) or G.L. (gxluep@wm.edu)

\author{
Wei Zheng ' ${ }^{1}$ Aubrey T. Hanbicki ${ }^{2}$, Berend T. Jonker ${ }^{2} \&$ Gunter Lüpke'
}

'Department of Applied Science, College of William \& Mary, Williamsburg, VA 23187, USA, ${ }^{2}$ Materials Science \& Technology Division, Naval Research Laboratory, Washington, D.C. 20375, USA.

The interaction between surface plasmons (SP) and magnetic behavior has generated great research interest due to its potential for future magneto-optical devices with ultra-high sensitivity and ultra-fast switching. Here we combine two surface sensitive effects: magnetic second-harmonic generation (MSHG) and SP to enhance the detection sensitivity of the surface magnetization in a single-crystal iron film. We show that the MSHG signal can be significantly enhanced by SP in an attenuated total reflection (ATR) condition, and that the magnetic contrast can be varied over a wide range by the angle-of-incidence. Furthermore, the magnetic contrast of transverse and longitudinal MSHG display opposite trends, which originates from the change of relative phase between MSHG components. This new effect enhances the sensing of magnetic switching, which has potential usage in quaternary magnetic storage systems and bio-chemical sensors due to its very high surface sensitivity and simple structure.

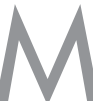

agneto-plasmonics (MP) describes hybrid systems where plasmonics and magnetic properties coexist, and has great potential for magneto-optical $(\mathrm{MO})$ devices ${ }^{1}$. The magnetic contrast plays an important role in MP systems and can be defined as $C=[I(+M)-I(-M)] /[I(+M)+I(-M)]$, where $I(+M)$ and $I(-M)$ are intensities for the two magnetization states. For example, in an active MP system, the modulation of surface plasmon wave vector can be expressed as a function of magnetic contrast ${ }^{2}$; in a new generation of biochemical sensing devices based on MP effect, the enhancement of sensitivity is a direct result of the reflection shift induced by the magnetic contrast ${ }^{3-6}$; and in more general cases, a high magnetic contrast is desirable for MO device applications because of the better signal-to-noise ratio revealing strong coupling between light and magnetic property. In this article, we demonstrate a large variation of magnetic contrast with nonlinear magneto-plasmonics (NMP). Here NMP refers to the nonlinear optical response from a MP system.

As a surface sensitive magneto-optical effect, magnetic second-harmonic generation (MSHG) is sensitive to the interface or surface magnetization where the inversion symmetry is broken. MSHG contains nonzero magnetic components of the second-order nonlinear susceptibility tensor which makes MSHG extremely sensitive to subtle modifications of the spin-polarized electronic structure of transition metal surfaces ${ }^{7-11}$, the same region where surface plasmons (SP) are present. The surface sensitivity of MSHG is different from the bulk effect of magnetooptical Kerr effect (MOKE) $)^{12,13}$, which is often used in MP systems. Historically, one dilemma for MP systems is that the noble metal layer supports high quality SP, but a very large magnetic field (several Tesla) is needed to achieve MO activity. On the other hand, the ferromagnetic (FM) layer exhibits large MO activity, which makes it possible to make real MO devices, but the high losses in the FM layer causes an over-damping of plasmon resonance, and thus impairs the strength of $\mathrm{SP}^{14,15}$. The prevailing configuration, noble metal/FM/noble metal heterostructures ${ }^{2-6,10-12}$, combines the strength of good quality SP and large MO effect, but makes the structure complex and separates the source of SP and magnetization which compromises the coupling between them. The unique feature of NMP based on MSHG technique is the combination of the two surface sensitive effects, which makes it of interest for bio-chemical sensors.

We note here that work performed by Razdolski et al. ${ }^{16}$ showed an enhanced contrast ratio of transverse MSHG by surface plasmon. The authors attributed this enhancement to a change in phase difference between magnetic and non-magnetic responses. In this article, we find that the contrast ratios of transverse and longitudinal MSHG exhibit different trends when changing the angle-of-incidence. Moreover, we clearly separate the L- and Tcomponent by studying the anisotropy and the two-jump hysteresis loops from a single-crystal Fe film, while others did not. We developed formulas for the two-jump switching loop to extract the ratios of magnitude and the phase differences between magnetic and non-magnetic components for both L- and T-MSHG, while only the longitudinal one has been studied previously ${ }^{11}$. This new effect enhances the sensing of magnetic switching, which has potential usage in quaternary magnetic storage systems because it enables the read-out of all four magnet- 
ization states from crystalline iron with high contrast ratio, and it is also of interest for bio-chemical sensor applications due to its very high surface sensitivity and simple structure.

Here, all experiments are carried out on single-crystalline iron films grown on $\mathrm{MgO}$ (001) substrates ${ }^{17}$ (see Supplementary Information - sample growth and characterization), which show unique magnetic switching behavior due to cubic magneto-crystalline anisotropy. The two-step switching characteristic of the Fe film generated by its cubic magnetic anisotropy ${ }^{18-20}$ (Fig. 1) allows us to simultaneously study both longitudinal (L) and transverse (T) component for MSHG and MOKE. The L-component corresponds to the magnetization $(\mathrm{M})$ component parallel to the plane-of-incidence, i.e. along the axis $[-110]$, while T-component is perpendicular ${ }^{7}$, i.e. along the axis [110]. The magnetization of a thin Fe film is always in the sample plane if the external magnetic field $(\mathrm{H})$ is applied parallel to the surface. The cubic magnetic anisotropy of single-crystal iron leads to two easy axes ([100] and [010]) and two hard axes ([110] and $[-110])$, and the magnetization tends to align along the easy axes (Fig. 1). Beginning from an initial state $M_{I}$ along [100], with increasing magnetic field along $[-110]$, the magnetization overcomes the hard axis [110], which causes a change in the longitudinal magnetic component $\left(\mathrm{M}_{\mathrm{I}}\right.$ to $\left.\mathrm{M}_{\mathrm{II}}\right)$. As $\mathrm{H}$ increases further, the magnetization overcomes another hard axis $[-110]$, which causes a change in the transverse magnetic component $\left(\mathrm{M}_{\mathrm{II}}\right.$ to $\left.\mathrm{M}_{\mathrm{III}}\right)$. We note that the jumps from $\mathrm{M}_{\mathrm{II}}$ to $\mathrm{M}_{\mathrm{III}}$ and $\mathrm{M}_{\mathrm{IV}}$ to $\mathrm{M}_{\mathrm{I}}$ are possible because the external magnetic field can never be perfectly parallel to the hard axis [-110], and a slight mis-orientation can cause such jumps. In the MSHG and MOKE hysteresis loops, each magnetization state corresponds to different signal intensity, I(M). The L-jump occurs at low coercivity while the T-jump happens at higher coercivity, because $\mathrm{H}$ is applied along the longitudinal direction and only a small field is needed to switch the L-component. From the two-jump hysteresis loops the L- and T-components can be separated clearly and studied simultaneously.

\section{Results}

We use the Kretschmann-Raether configuration ${ }^{21}$ to excite the SP in our experiments (Fig. 2a). For a P-polarized incident beam, the reflection is maximized when the total reflection angle $\left(\theta=40^{\circ}\right)$ is reached. The reflection decreases sharply when the incident angle increases further, which is called attenuated total reflection (ATR). The ATR curve can be explained by the coupling of light energy into the SP on the metal surface. The intensity of the electro-magnetic field on the metal surface at the metal-air interface becomes enhanced under the ATR condition, and reaches its maximum at the bottom of the ATR curve for noble metals ${ }^{22,23}$. For iron, the ATR curve is stretched out due to absorption, i.e., strong damping (Fig. 2b).

Figure $2 \mathrm{~b}$ shows an enhancement of MSHG intensity by SP of more than one order of magnitude. In addition, the magnetic contrast becomes very large under ATR condition. Note that the SPenhanced MSHG at the metal/air interface is much greater than that at the substrate/metal interface ${ }^{24}$, so that $\mathrm{MSHG}$ at the $\mathrm{MgO}-\mathrm{Fe}$ interface is not being considered. For iron, the width of ATR is very large and the difference of incident angle for highest field strength and minimum of ATR curve is also large. As shown in reference 24, the maximum of the MSHG signal does not coincide with the highest field strength.

Figure 2c shows MSHG hysteresis loops taken at different incident angles, $\theta=41^{\circ}, 41.5^{\circ}, 47.5^{\circ}$, and $52.5^{\circ}$. There is no magnetic contrast at the angle of total reflection $\left(\theta=40^{\circ}\right)$. For $\theta=41^{\circ}$ ATR just begins, the hysteresis loop shows clear contrast with only the L-component switching at $\mathrm{H}=5 \mathrm{Oe}$. The switching of T-component at $\mathrm{H}=40 \mathrm{Oe}$ begins to appear at $\theta=41.5^{\circ}$, but the L-component still dominates the switching. With increasing incident angle $\left(\theta=47.5^{\circ}\right)$, the $\mathrm{T}$ component becomes more enhanced, and eventually by $\theta=52.5^{\circ}$, where the reflection is very small and close to the bottom of the ATR curve, the T-component dominates the hysteresis loop. For comparison, Fig. $2 \mathrm{~d}$ shows hysteresis loops for SP-enhanced MOKE. At $\theta=$ $41^{\circ}$ and $41.5^{\circ}$, the T-component is negligible. At larger angles, $\theta=$ $47.5^{\circ}$ and $\theta=52.5^{\circ}$, the $\mathrm{T}$-component becomes enhanced but remains at a relatively low level as compared to the L-component. This important observation demonstrates the large variation of Land T-component in MSHG, which is absent in MOKE. There is no such huge effect for MSHG under normal reflection geometry or MSHG under Kretschmann-Raether configuration with S-polarized fundamental field. In the following, we elucidate the origin of the observed effect in terms of magnetic contrast.

The magnetic contrast for a one-jump hysteresis loop can be expressed $\mathrm{as}^{7}$ :

$$
C=\frac{I(+M)-I(-M)}{I(+M)+I(-M)},
$$

where $I(+M)$ and $I(-M)$ are intensities for the two magnetization states. The magnetic contrast can be understood as the height of the jump in the hysteresis loop divided by the average intensity of both

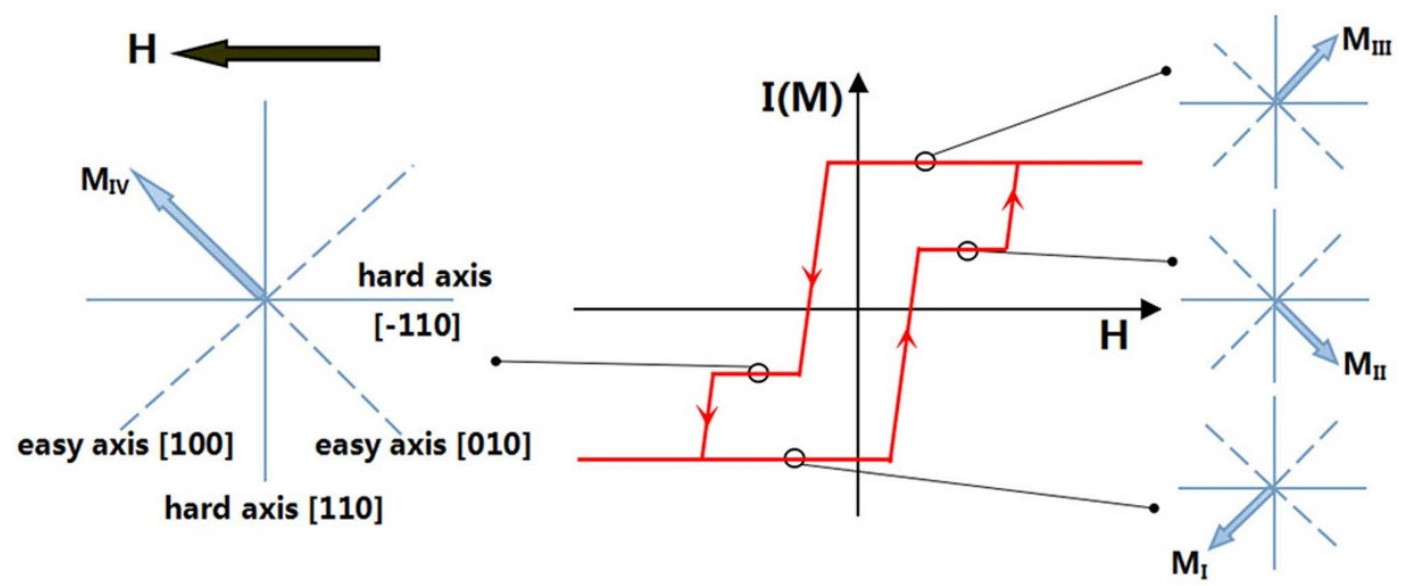

Figure $1 \mid$ Crystalline magnetic anisotropy and two-jump switching. A magnetically anisotropic material like Fe will align its magnetic moment with one of the easy axes ([100] and [010]), which are energetically favorable directions. A very small external field $\mathrm{H}$ applied along the longitudinal direction, i.e. the hard axis [-110], can switch the magnetization from $M_{I}$ to $M_{I I}$ because $H$ is applied along the jump direction (L-jump). On the other hand, a much larger field is required to switch $\mathrm{M}$ transverse to the direction of $\mathrm{H}$ (T-jump), i.e., from $\mathrm{M}_{\mathrm{II}}$ to $\mathrm{M}_{\mathrm{III}}$. Each magnetization state corresponds to different signal intensity, $\mathrm{I}(\mathrm{M})$, in the hysteresis loop. 
a

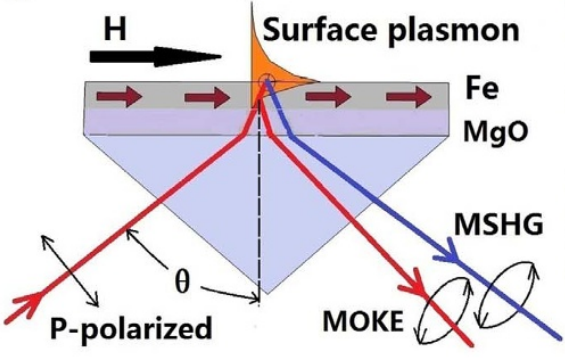

C

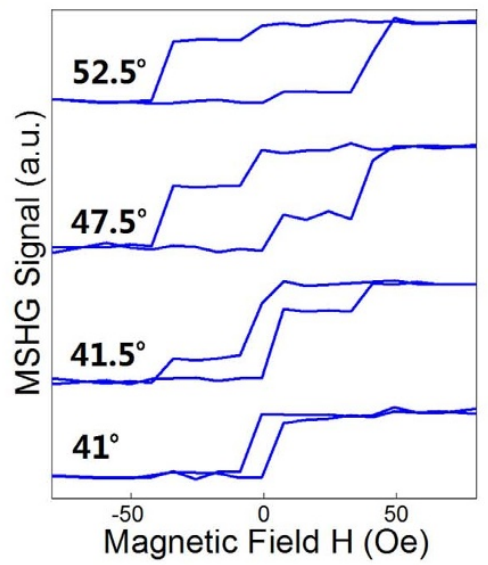

b

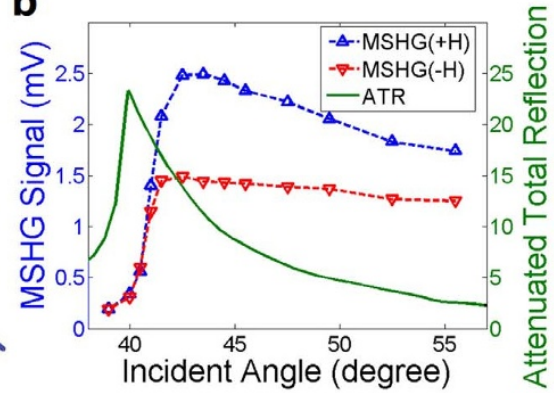

d

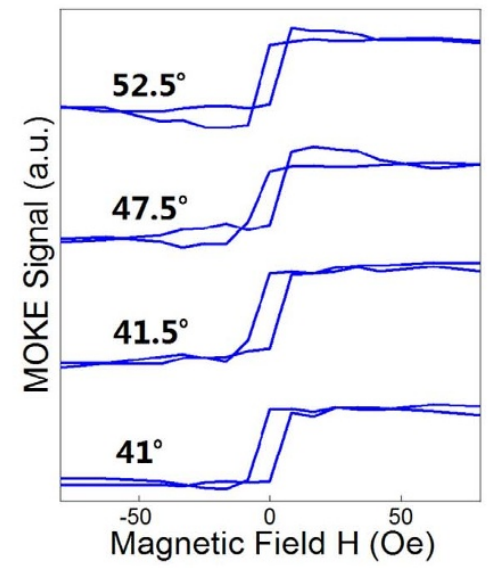

Figure $2 \mid$ Schematic of Nonlinear Magneto-Plasmonics, ATR curve, and MSHG and MOKE hysteresis loops. (a), Experimental configuration for surface plasmon excitation and detection of MSHG and MOKE by ATR configuration, where $\theta$ is the incident angle and the external magnetic field $\mathrm{H}$ is applied along the longitudinal direction, i.e. the hard axis [-110]. (b), ATR and SP-enhanced MSHG curves. (c), MSHG hysteresis loops. The Tcomponent increases with incident angle until it dominates the switching process, while the L-component decreases. (d), MOKE hysteresis loops. The Tcomponent remains small even for very large incident angles, while the L-component remains mostly unchanged.

magnetizations. In the same way, we define the T- and L- magnetic contrast for two-jump hysteresis loop as (see Supplementary Information: Magnetic contrast of two-jump hysteresis loop):

$$
\begin{aligned}
& C_{T}=\frac{\left(I\left(M_{I I}\right)-I\left(M_{I I}\right)\right)+\left(I\left(M_{I V}\right)-I\left(M_{I}\right)\right)}{I\left(M_{I}\right)+I\left(M_{I I}\right)+I\left(M_{I I I}\right)+I\left(M_{I V}\right)} \text { and } \\
& C_{L}=\frac{\left(I\left(M_{I I I}\right)-I\left(M_{I V}\right)\right)+\left(I\left(M_{I I}\right)-I\left(M_{I}\right)\right)}{I\left(M_{I}\right)+I\left(M_{I I}\right)+I\left(M_{I I I}\right)+I\left(M_{I V}\right)},
\end{aligned}
$$

where $M_{I}, M_{I I}, M_{I I}$ and $M_{I V}$ are depicted in Fig. 1 . We note that $C_{L}$ and $C_{T}$ are directly related to the direction of the magnetization, rather than the external magnetic field. This means that the same contrast ratios are obtained when the external magnetic field $\mathrm{H}$ is applied along the longitudinal or transverse direction, i.e. hard axis $[-110]$ or $[110]$.

Figure 3 shows the magnetic contrast of MSHG and MOKE as a function of the incident angle, together with the ATR curve with $\mathrm{H}$ applied along the transverse direction, i.e. the hard axis [110]. The most important finding is that for MSHG, $C_{L}\left(\boldsymbol{\sim}()\right.$ ) and $C_{T}(\neg-)$ can be varied by the SP resonance with opposite trend. We call this control of magnetic contrast with nonlinear magneto-plasmonics. $C_{L}$ initially increases sharply when ATR begins and reaches its maximum at around $42.5^{\circ}$, but then decreases as $\theta$ increases further, until it totally disappears at around $55^{\circ}$. In contrast, $C_{T}$ increases slowly and steadily over the whole range. This trend is independent of the setting of analyzer.

For MOKE, $C_{T}(\triangleleft-\cdot)$ qualitatively follows the same trend as the MSHG, but the SP field enhancement is not nearly as strong because MOKE is a bulk effect. For longitudinal MOKE, $C_{L}\left(-\downarrow^{-}\right)$shows only a small change as a function of the angle-of-incidence. The small minimum which occurs where $C_{L}$ (MSHG) reaches a maximum indicates a small SP field enhancement in the longitudinal MOKE which interferes destructively with the bulk MOKE signal.

\section{Discussion}

To elucidate the origin of the large variation in the MSHG contrast ratios, we studied $C_{T}$ and $C_{L}$ as a function of the angular position $\alpha$ of

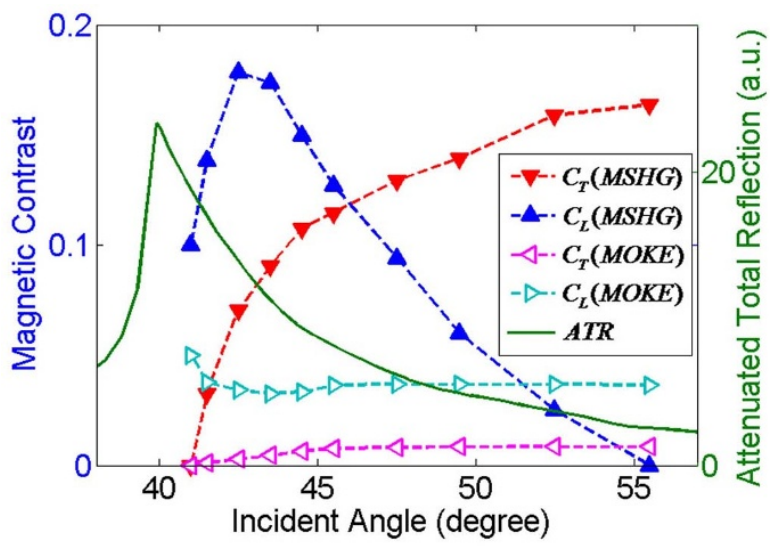

Figure 3 Magnetic contrast of MSHG and MOKE under ATR. For MSHG, the magnetic contrast for the L-component initially increases but then decreases as the incident angle increases, while the T-component steadily increases. In contrast, for MOKE the L-component does not change much over the total reflection, and the enhancement of the Tcomponent is also limited. The external magnetic field $\mathrm{H}$ is applied along the transverse direction, i.e. the hard axis [110]. 


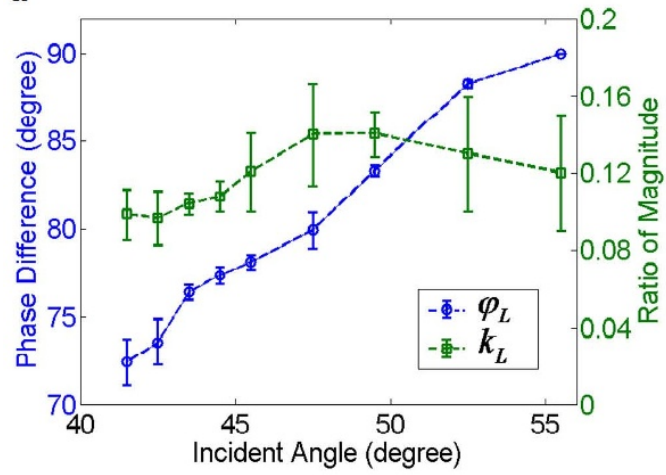

b

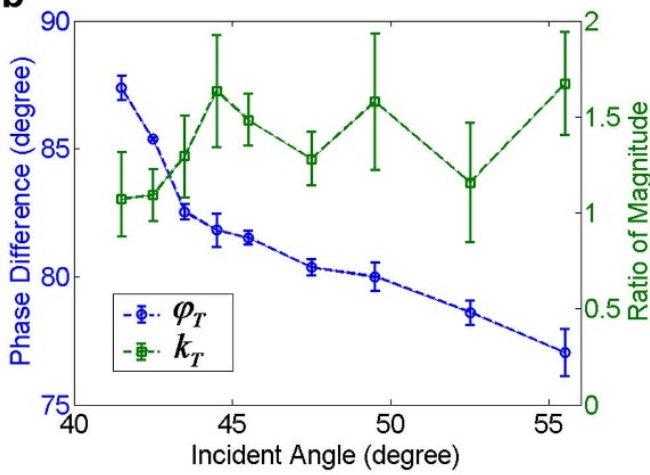

Figure $4 \mid$ Phase difference and ratio of magnitude as a function of incident angle. (a), L-MSHG. (b), T-MSHG. The phase differences exhibit opposite trends for L- and T- magnetic components, while the ratios of magnitude remain unchanged within experimental errors. The external magnetic field $\mathrm{H}$ is applied along the transverse direction, i.e. the hard axis [110].

the analyzer ${ }^{11,25}(\alpha=0$ corresponds to p-polarization). The magnetic contrasts can be expressed as [see Supplementary Information for details]:

$$
C_{L}=2 \frac{k_{L} \tan (\alpha) \cos \left(\varphi_{L}\right)}{1+k_{T}^{2}+k_{L}^{2} \tan ^{2}(\alpha)} \text { and } C_{T}=2 \frac{k_{T} \cos \left(\varphi_{T}\right)}{1+k_{T}^{2}+k_{L}^{2} \tan ^{2}(\alpha)}
$$

where $k_{T}$ and $k_{L}$ are the ratios of magnitude between the magnetic and non-magnetic MSHG response for T- and L-magnetization components, both of which are composed of fundamental fields and corresponding effective susceptibility tensors. $\varphi_{T}$ and $\varphi_{L}$ are the phase differences between the magnetic and non-magnetic MSHG response for T- and L-magnetization components, respectively. We note that when $k_{T}=0$, the expression for $C_{L}$ becomes identical to the one for the one-jump MSHG hysteresis loop measured in the L-geometry. Furthermore, $k_{T}$ and $\varphi_{T}$ can only be obtained from the two-jump MSHG hysteresis loop, because Eq. (3) becomes meaningless without $k_{L}$ and $\alpha$.

Figures $4 \mathrm{a}$ and $4 \mathrm{~b}$ show the ratios of magnitude and phase differences as a function of incident angle under ATR condition for T- and L-magnetization components, respectively. The ratios $k_{T}$ and $k_{L}$ hardly change within experimental errors and hence cannot account for the large variation. In contrast, $\varphi_{L}$ exhibits a large monotonic increase from $72^{\circ}$ to $90^{\circ}$ over the ATR range, while $\varphi_{T}$ decreases from $88^{\circ}$ to $77^{\circ}$. This trend is consistent with the large decrease of $C_{L}$ and the steady increase of $C_{T}$ with increasing incident angle under ATR. Therefore, the large variation of magnetic contrast originates from the change of relative phases caused by SP. The phase difference between magnetic and non-magnetic components of MSHG signal is known to be related to the relative orientation of magnetization and the wave vector of fundamental fields ${ }^{16,26}$. Since the L- and T- magnetization components are normal to each other, $C_{T}$ and $C_{L}$ display opposite trends.

In summary, we have demonstrated control of magnetic contrast in single-crystalline iron film with nonlinear magneto-plasmonics. Such a system opens the way for simultaneously investigating both longitudinal and transverse magnetization components regardless of the external magnetic field. By studying MSHG hysteresis loops as a function of incident angle and polarization angle, and by fitting the magnetic contrasts to theoretical formulas, we find that the observed effect originates from the change of relative phase between magnetic and non-magnetic MSHG components caused by surface plasmons. This new effect has potential applications in quaternary magnetic storage systems and is also of interest for bio-chemical sensor applications.

1. Temnov, V. V. Ultrafast acousto-magneto-plasmonics. Nature Photon. 6, 728-736 (2012).
2. Temnov, V. V. et al. Active magneto-plasmonics in hybrid metal-ferromagnet structures. Nature Photon. 4, 107-110 (2010).

3. Armelles, G., Cebollada, A., Garcia-Martin, A. \& González, M. Magnetoplasmonics: Combining magnetic and plasmonic functionalities. $A d v$. Opt. Mat. 1, 10-35 (2013).

4. Pistora, J., Lesnak, M. \& Vlasin, O. Surface plasmon resonance sensor with Magneto-Optical thin film. Acta Electrotechnica et Informatica 10, 19-21 (2010).

5. Sepúlveda, B., Calle, A., Lechuga, L. M. \& Armelles, G. Highly sensitive detection of biomolecules with the magneto-optic surface-plasmon-resonance sensor. Opt. Lett. 31, 1085-1087 (2006).

6. Regatos, D. et al. Au/Fe/Au multilayer transducers for magneto-optic surface plasmon resonance sensing. J. Appl. Phys. 108, 054502 (2010).

7. Kirilyuk, A. \& Rasing,T. Magnetization-induced second harmonic generation. Handbook of Magnetism and Advanced Magnetic Materials. Volume 3: Novel Techniques for Characterizing and Preparing Samples. (John Wiley \& Sons, Ltd. 2007).

8. Fiebig, M., Fröhlich, D., Krichevtsov, B. B. \& Pisarev, R. V. Second harmonic generation and magnetic-dipole electric-dipole interference in antiferromagnetic $\mathrm{Cr}_{2} \mathrm{O}_{3}$. Phys. Rev. Lett. 73, 2127-2130 (1994).

9. Zhao, H. B. et al. Interface Magnetization Reversal and Anisotropy in Fe/AlGaAs(001). Phys. Rev. Lett. 95, 137202 (2005).

10. Valev, V. K. et al. Plasmons reveal the direction of magnetization in nickel nanostructures. ACS Nano 5, 91-96 (2011).

11. Tessier, G. et al. Magnetization-induced second-harmonic generation enhanced by surface plasmons in ultrathin $\mathrm{Au} / \mathrm{Co} / \mathrm{Au}$ metallic films. Appl. Phys. B 68, 545-548 (1999).

12. Safarov, V. I. et al. Magneto-optical effec][ts enhanced by surface plasmons in metallic multilayer films. Phys. Rev. Lett. 73, 3584-3587 (1994).

13. Maruyama, T. et al. Large voltage-induced magnetic anisotropy change in a few atomic layers of iron. Nature Nanotech. 4, 158-161 (2009).

14. Melander, E. et al. Influence of the magnetic field on the plasmonic properties of transparent Ni anti-dot arrays. Appl. Phys. Lett. 101, 063107 (2012).

15. Bonanni, V. et al. Designer magnetoplasmonics with nickel nanoferromagnets. Nano Lett. 11, 5333-8 (2011).

16. Razdolski, I. et al. Nonlocal nonlinear magneto-optical response of a magnetoplasmonic crystal. Phys. Rev. B 88, 075436 (2013).

17. Fan, Y. et al. Exchange bias of the interface spin system at the Fe/MgO interface. Nature Nanotech. 10, 1038-1043 (2013).

18. Daboo, C. et al. Magnetization reversal processes in epitaxial Fe/GaAs(001) films. App. Phys. Lett. 75, 5586 (1994).

19. Cowburn, R. P. et al. Magnetic switching and in-plane uniaxial anisotropy in ultrathin Ag/Fe/Ag(100) epitaxial films. App. Phys. Lett. 78, 7210 (1995).

20. Daboo, C. et al. Anisotropy and orientational dependence of magnetization reversal processes in epitaxial ferromagnetic thin films. Phys. Rev. B 51, 15964 (1999).

21. Raether, H. Surface Plasmons on Smooth and Rough Surfaces and on Gratings. (Springer Tracts in Modern Physics, 1988).

22. Simon, H. J., Mitchell, D. E. \& Watson, J. G. Optical Second-Harmonic Generation with Surface Plasmons in Silver Films. Phys. Rev. Lett. 33, 1531-1534 (1974).

23. Weber, W. H. \& Ford, G. W. Optical electric-field enhancement at a metal surface arising from surface-plasmon excitation. Opt. Lett. 6, 122-124 (1981).

24. Zheng, W., Hanbicki, A. T., Jonker, B. T. \& Lüpke, G. Surface plasmon-enhanced transverse magnetic second-harmonic generation. Opt. Exp. 21, 28842-28848 (2013).

25. Koopmans, B., Koerkamp, M. G. \& Rasing, Th. Observation of large Kerr angles in the nonlinear optical response from magnetic multilayers. Phys. Rev. Lett. 74, 3692-3695 (1995). 
26. Spierings, G. et al. Interface magnetism studied by optical second harmonic generation. J. Magn. Magn. Mater. 121, 109-111 (1993).

\section{Acknowledgments}

The optical experiments performed at the College of William and Mary are supported by the Department of Energy through Grant No. DE-FG02-04ER46127. The sample growth at NRL is supported by core programs and the Office of Naval Research.

\section{Author contributions}

W.Z. and G.L. conceived and designed the experiment, A.T.H. and B.J. fabricated the samples, W.Z. acquired the optical data, W.Z. and G.L. analyzed the data, all authors discussed and contributed to the interpretation and writing the paper. G.L. supervised the research.

\section{Additional information}

Supplementary information accompanies this paper at http://www.nature.com/ scientificreports

Competing financial interests: The authors declare no competing financial interests. How to cite this article: Zheng, W., Hanbicki, A.T., Jonker, B.T. \& Lüpke, G. Control of magnetic contrast with nonlinear magneto-plasmonics. Sci. Rep. 4, 6191; DOI:10.1038/ srep06191 (2014).

(c) (1) () $\odot$ This work is licensed under a Creative Commons Attribution-NonCommercialNoDerivs 4.0 International License. The images or other third party material in this article are included in the article's Creative Commons license, unless indicated otherwise in the credit line; if the material is not included under the Creative Commons license, users will need to obtain permission from the license holder in order to reproduce the material. To view a copy of this license, visit http:// creativecommons.org/licenses/by-nc-nd/4.0/ 